\title{
Effects of Lower Rib Expansion Limitation on Maximal Respiratory Pressure and Abdominal Muscle Activity During Maximal Breathing in Healthy Subjects
}

\author{
Gyu-wan Lee', Tae-Lim Yoon², Young-jung Lee ${ }^{3}$, Ki-song Kim4, Chung-hwi Yi ${ }^{5}$ \\ 'Department of Physical Therapy, Gangnam Severance Hospital, Seoul, Republic of Korea; ${ }^{2}$ Department of Physical Therapy, College of Health and \\ Medical Science, Cheongju University, Cheongju, Republic of Korea; ${ }^{3}$ Department of Physical Therapy, Bundang Jesaeng Hospital, Seongnam, \\ Republic of Korea; ${ }^{4}$ Department of Physical Therapy, College of Natural Science, Hoseo University, Asan, Republic of Korea; ${ }^{5}$ Department of Physical \\ Therapy, College of Health Science, Yonsei University, Wonju, Republic of Korea
}

Purpose: The aim of this study was to determine the effects of lower rib cage lateral expansion limitation on the maximal inspiratory and expiratory pressures and on abdominal muscle activity during maximal respiratory breathing in healthy subjects.

Methods: Fifteen healthy male subjects voluntarily participated in this cross-sectional study. During maximal breathing, maximal inspiratory and expiratory pressures were measured, and abdominal muscle activity was determined with using surface electromyography. Also, the measurement was repeated with using a non-elastic belt to the lower rib cage for limiting of lateral expansion. A Wilcoxon signed-rank test was performed for obtaining the statistical difference with a significance level of 0.05 .

Results: The findings of this study are as follows: 1) There were no significant differences in maximal inspiratory and expiratory pressure with and without lower rib cage lateral expansion ( $p>0.05)$, 2) There was no significant difference in abdominal muscle activity during the maximal inspiratory phase ( $p>0.05$ ). However, right external oblique muscle activity decreased significantly during maximum exhalation with lower rib expansion limitation $(p<0.05)$.

Conclusion: The results of the current study indicate that a non-elastic belt was effective in decreasing right external oblique muscle activity during forced expiratory breathing in healthy subjects.

Key Words: Abdominal muscle activity, Maximal inspiratory and expiratory pressure, Respiratory pressure meter

\section{INTRODUCTION}

The diaphragm, transverse abdominalis, pelvic floor muscles, and multifidus muscles, which play an important role in the function of postural control, are representative spinal stabilization muscles. ${ }^{1-3}$ In particular, the diaphragm plays an important role in stabilizing the spine when performing tasks with adequate consideration of balance and load application. ${ }^{4,5}$ De Troyer and Estennel described the cylindrical area directly enclosed by the inner surface of the lower rib as the zone of apposition (ZOA). If the curvature of this area is decreased or inappropriate, insufficient breathing occurs due to a decrease in transdiaphragmatic pressure, and the activity of the transverse abdominalis on spinal stabilization and breathing decreases. ${ }^{6,7}$ Eventually, as the stability of the lower ribs decreases, the role of

Received Dec 9, 2020 Revised Dec 31, 2020

Accepted Dec 31, 2020

Corresponding author Chung-hwi Yi

E-mail free0829@gmail.com the diaphragm and the activity of the abdominal muscles also decrease. ${ }^{8}$ The activity of the abdominal muscles is usually confirmed by electromyography, but it is difficult to directly measure the muscle activity of the diaphragm. Therefore, in order to indirectly determine the strength of the respiratory muscles, including the diaphragm, the maximal respiratory pressure is measured. ${ }^{9} 10$

Low back pain was confirmed to have a higher correlation with respiratory disease than obesity and physical activity. ${ }^{11}$ In addition, in patients with chronic low back pain, the excursion of the diaphragm was decreased compared to that of a normal person, and excessive diaphragmatic muscle recruitment was required due to the abnormally high position of the diaphragm. ${ }^{12}$ Perri and Halford ${ }^{12}$ reported a correlation between chronic musculoskeletal pain and abnormal breathing. Abnormal breathing pat-

Copylight (C)2020 The Korean Society of Physical Therapy

This is an Open Access article distribute under the terms of the Creative Commons Attribution Non-commercial License (https:// creativecommons.org/license/by-nc/4.o.) which permits unrestricted non-commercial use, distribution, and reproduction in any medium, provided the original work is properly cited. 
terns include excessive movement of the rib cage, elevation of the upper rib cage during inspiration, loss of or lifting during the lateral movement of the ribs, lack of abdominal movement, and paradoxical breathing. Janssens et al. ${ }^{14}$ reported that after inducing acute inspiratory muscle fatigue, inadequate proprioceptive postural control was seen in patients with low back pain compared to normal subjects using multisegmental postural control. This means that postural control is reduced in patients with low back pain. In addition, patients with low back pain reported significantly increased diaphragmatic fatigue after inspiratory muscle load than normal subjects. ${ }^{15}$ As such, in patients with low back pain, reduced diaphragmatic displacement, abnormal breathing patterns, and decreased postural control are also present.

Limiting lower rib expansion with a belt or brace affects the breathing pattern. Hussain and Pardy ${ }^{16}$ suggested that selective rib expansion restriction using a non-elastic corset in normal subjects alters breathing patterns during high-intensity exercise, reduces diaphragm contractility, and increases abdominal muscle recruitment during exhalation. Van Noord et al. ${ }^{17}$ reported that in normal subjects, the restriction of expansion by a belt in the ribs and abdominal area decreased the total lung volume, and increased the specific airway conductance and maximal expiratory flow. Excessive lower ribcage expansion and diaphragm shortening have been reported to shorten $\mathrm{ZOA}{ }^{18}$ In particular, this is clinically more prominent in patients with chronic low back pain.

No studies have been previously conducted to evaluate the effects of restriction of lower rib expansion on maximum respiratory pressure or abdominal muscle activity during respiration. However, before it was immediately applied to patients with low back pain, this study decided to conduct a study in the general population. Therefore, this study investigated the effect of limiting lower rib expansion using a non-elastic belt on maximum respiratory pressure and abdominal muscle activity during maximal respiration in healthy adults.

\section{METHOD}

\section{Subjects}

This study was conducted on 15 healthy adult males. The exclusion criteria were as follows: heart disease, pulmonary disease, musculoskeletal disease, abnormal curvature or deformation of the spine, neurological disease, neck pain, and low back pain. The subjects of this study voluntarily participated in the experiment, received detailed explanations about the experiment, and signed an experiment consent form. The general charac-
Table 1. General characteristics of subjects

$(\mathrm{N}=15)$

\begin{tabular}{lr}
\hline Characteristics & Mean \pm SD \\
\hline Age $(\mathrm{yr})$ & $20.6 \pm 2.1$ \\
Height $(\mathrm{cm})$ & $173.5 \pm 7.6$ \\
Weight $(\mathrm{kg})$ & $62.8 \pm 8.2$ \\
BMI $\left(\mathrm{kg} / \mathrm{m}^{2}\right)$ & $20.8 \pm 1.7$
\end{tabular}

mean \pm standard deviation, BMl: body mass index.

teristics of the study subjects are shown in Table 1.

\section{Measurements}

\section{1) Surface electromyography (sEMG)}

The muscle activity of the abdominal muscles was measured using Noraxon Telemyo 2004T (Noraxon Inc., Scottsdale, AZ, USA). The surface EMG analog signals collected from the rectus abdominis, external oblique, and internal oblique were converted into digital signals and processed using MyoResearch XP Master 1.06 software on a personal computer. The sampling rate of the EMG signals was set to $1,024 \mathrm{~Hz}$, the frequency bandwidth (bandpass-filtered) to $20-450 \mathrm{~Hz}$, and the notch filter to $60 \mathrm{~Hz}$. In order to minimize skin resistance when measuring EMG, hair was removed from the electrode attachment site before attaching the electrode; skin was exfoliated with sandpaper, and then cleaned with alcohol alcohol swabs. As for the electrodes, silver/silver chloride (Ag/AgCl) ones were attached parallel to the longitudinal direction of each muscle fiber with an interval of approximately $2 \mathrm{~cm}$. According to Criswell and $\mathrm{Cram}^{19}$, the rectus abdominis muscle is approximately $3 \mathrm{~cm}$ lateral to the navel, the oblique abdominal muscles are approximately $15 \mathrm{~cm}$ lateral to the navel, and the rectus abdominis muscle is just above the midpoint of the inguinal ligament between the upper anterior hip bone and the midline of the pelvis. Also, external oblique muscle (approximately $15 \mathrm{~cm}$ lateral to the umbilicus) and internal oblique muscle (the geometric center of the triangle formed by the inguinal ligament, the outer edge of the rectus sheath, and the imaginary line joining the anterior superior iliac spine and the umbilicus) are selected. ${ }^{20}$ After attaching the EMG, muscle activity was measured by performing maximal voluntary isometric contraction (MVIC) in the manual muscle strength test posture in order to normalize the EMG values. ${ }^{21}$ The maximum voluntary isometric contraction measurement was repeated three times for 5 seconds in each posture, and the EMG data for 5 seconds were treated as a root mean square (RMS), and the middle of the first and last, excluding 1 second each. The average EMG over 3 seconds was presented as \%MVIC. 


\section{2) Maximum respiratory pressure}

The maximum respiratory pressure is divided into the maximum expiratory pressure and the maximum inspiratory pressure. In addition, the strength of the respiratory muscles, including the diaphragm, can be used for an indirect evaluation. ${ }^{910}$ In this study, a portable maximum respiratory pressure meter (Respiratory Pressure Meter, Micro Medical Limited, Kent ME1 2AZ England) was used to measure the maximum respiratory pressure. This measuring instrument displays the maximum breathing pressure through a pressure transducer and an electronic calculator, and the result is reflected on a liquid crystal display. The maximum respiratory pressure was measured while the subject maintained maximum inhalation and maximum exhalation while sitting comfortably. Subjects were cautioned to prevent air leakage between the mouthpiece and mouth. Maximum inhalation and maximum exhalation were maintained for at least 1 second. ${ }^{22}$ The unit of pressure used was $\mathrm{mmHg}$.

\section{Procedure}

\section{1) Without lower rib expansion limitation}

Subjects were allowed to maintain a relaxed state by performing normal breathing several times while sitting comfortably in a chair without armrests. The maximum respiratory pressure was measured using a respiration-measuring device. Maximum inspiratory pressure and maximum expiratory pressure were measured randomly to avoid the effect of the measurement sequence. Maximum expiratory pressure and maximum inspiratory pressure refer to the strength of the expiratory and inspiratory muscles, respectively. ${ }^{10}$ The maximum inspiratory pressure was measured after the subject was seated directly in a chair, the nose plugged, and maximum inspiratory effort was continuously made through the mouthpiece after exhaling to the maximum. The maximum expiratory pressure was measured during the maximum exhalation effort made after inhaling as much as possible in the same position. Subjects were measured five times at 1-minute intervals and the highest maximum value was used. In addition, abdominal muscle activity was measured at the time of maximum respiration. Subsequent trials were executed if the difference in the EMG peak of the two MVICs was greater than 5\%. The trial with the highest EMG peak was selected for analysis.

\section{2) With lower rib expansion limitation}

In order to limit the expansion of the lower rib cage, non-elastic belt (COM-PRESSORTM, OPTP, Minneapolis, USA), and applied to the lower rib and sternal area within the range that did not interfere with the
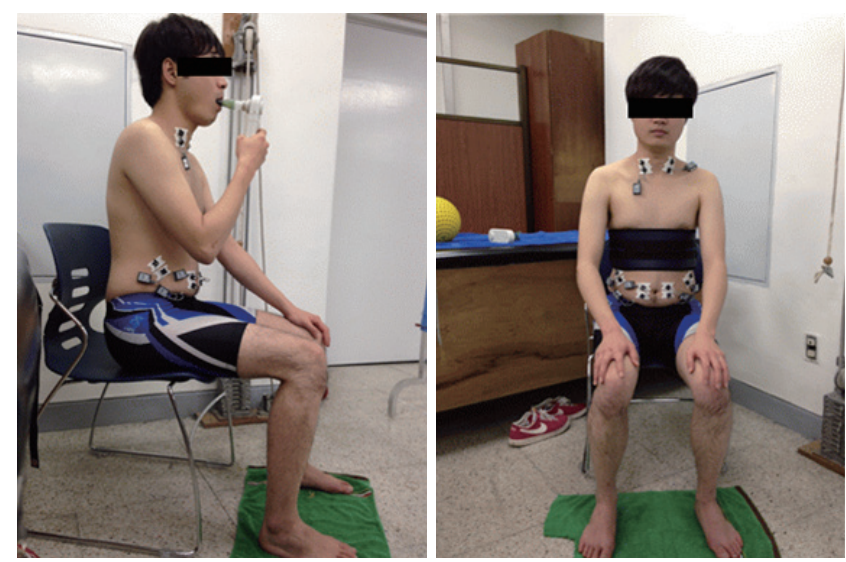

Figure 1. Maximal respiratory pressure and abdominal muscle activity with and without belt.

EMG electrodes. The non-elastic belt was applied to the lower rib (8-12) and sternum in a comfortable sitting position, and positioned in a way that it did not interfere with the EMG electrodes (Figure 1). ${ }^{23}$ After adjusting the pressure of the non-elastic belt to the extent that it did not interfere with normal exhalation and inhalation, breathing was performed several times as usual. The dependent variable, measured in the absence of lower rib expansion limitation, was measured in the previous method.

\section{Statistical analysis}

The Kolmogorov-Smirnov test was performed to check the normal distribution. Since the data didn't show the normal distribution with Kolmogorov-Smirnov test, the maximum respiratory pressure and the abdominal activity during maximum breathing were compared using the Wilcoxon signed rank test, which is a nonparametric statistical method. The analysis was performed using SPSS ver. 18.0 program, and the significance level was set to 0.05 .

\section{RESULTS}

\section{Comparison of the maximum respiratory pressures with and without lower rib expansion limitation:}

There was no statistically significant difference between the maximum inspiratory pressure (without belt $=74.0 \pm 22.9 \mathrm{mmHg}$, with belt $=78.4 \pm 22.6$ $\mathrm{mmHg}, \mathrm{p}=0.245$ ) and the maximum expiratory pressure (without belt $=$ $80.0 \pm 23.2 \mathrm{mmHg}$, with belt $=82.5 \pm 25.9 \mathrm{mmHg}, \mathrm{p}=0.201$ ) according to the presence or absence of lower rib expansion limitation $(\mathrm{p}>0.05)$. 


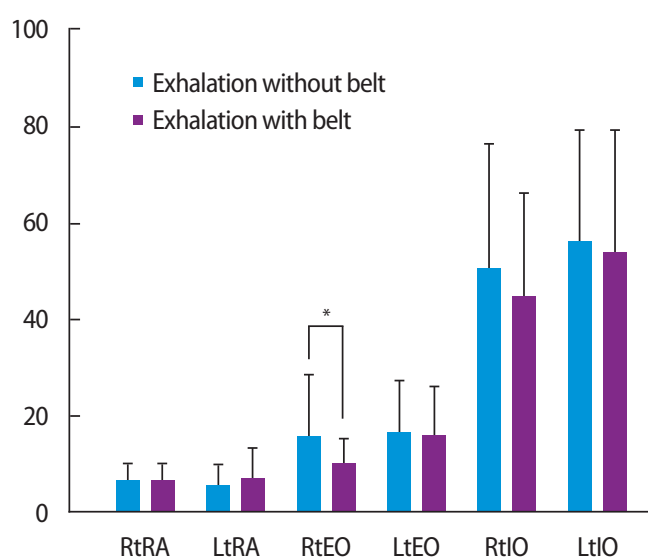

Figure 2. Maximum exhalation with and without belt. RA: rectus abdominis, EO: external oblique abdominis, IO: internal oblique abdominis, Data were presented with \% maximal voluntary isometrics contraction.

\section{Comparison of abdominal muscle activity during maximum breathing with and without lower rib expansion limitation:}

There was no significant difference in abdominal activity during maximum inspiration in relation to the presence or absence of lower rib expansion limitation $(\mathrm{p}>0.05)$. Figure 2 shows the results of abdominal muscle activity during maximum exhalation in relation to the presence or absence of thoracic mobility. When lateral expansion of the lower rib cage was restricted with belt, the right external oblique muscle activity decreased significantly during maximum exhalation $(\mathrm{p}<0.05)$.

\section{DISCUSSION}

The purpose of this study was to investigate the effects of limiting lower ribcage expansion using a non-elastic belt on the maximal inspiratory/expiratory pressures and the abdominal muscle activity during maximal breathing in normal subjects prior to a study on patients. There was no statistically significant difference in maximal respiratory pressure during maximal breathing, with and without lower rib expansion limitation. There was no significant difference in abdominal activity during maximal inspiration, with and without lower rib expansion limitation. However, the right external oblique muscle activity decreased significantly during maximum exhalation without lower rib expansion limitation.

There was no significant difference in the maximal inspiratory and expiratory pressures, with and without lower rib expansion limitation in healthy subjects. The investigator originally predicted that the maximal inspiratory pressure would increase significantly when the non-elastic belt was applied to the subject's lower rib cage to limit the lateral expansion of the lower rib cage during inspiration. The reason was that during inspiration, the pressure in the lungs decreases from the atmospheric pressure due to the descent of the contracting diaphragm, and due to the rise and expansion of the ribcage due to the contraction of the external intercostal muscles. Although this study did not directly measure diaphragmatic descent during inspiration, the reliability and validity of the maximal inspiratory pressure as an indirect indicator for evaluating descent due to diaphragmatic contraction has been demonstrated in previous studies. ${ }^{24,25}$

The results of this study could not conclude that when lower rib expansion was restricted, the diaphragmatic descent increased, which could further increase the maximal inspiratory pressure of air entering the lungs. This is because the pressure in the lungs decreases below atmospheric pressure from the lowering and contraction of the diaphragm that occurs when the subject inhales, and from the rise and expansion of the rib cage due to the contraction of the external intercostal muscles. ${ }^{1,26}$ It was expected that if lower thoracic expansion was restricted, the contraction of the outer intercostal space would be restricted to further promote the lowering of the diaphragm during inspiration, which would increase the maximal inspiratory pressure. The different unexpected result was thought to be due to the participation of young and healthy men who could perform inhalation without difficulty, even if thoracic expansion was limited. In particular, the male subjects mainly had a breathing pattern that contracted the diaphragm rather than expanding the chest, so it is thought that the limit of thoracic expansion, the independent variable of this study, had less influence on the breathing parameters. ${ }^{27}$ There was also no significant difference in the maximal expiratory pressure. There was no significant difference in the maximum inspiratory pressure with or without thoracic expansion restriction. There was no significant difference in the amount of inhaled air, so it was judged that the presence or absence of chest expansion restriction did not significantly affect exhalation. In further studies, if we examine the effect of thoracic expansion restriction by using a nonelastic belt in patients with decreased inspiratory or expiratory function, female subjects, or in patients with excessive thoracic expansion, significant clinical conclusions.

The results showed that there was no significant difference in abdominal activity at maximal inspiration in relation to the presence or absence of lower rib expansion limitation, but during maximal expiration, thoracic expansion limitation significantly decreased right external oblique muscle activity. In general, during stable exhalation, exhalation occurs due to an increase in pressure in the lungs due to passive relaxation. ${ }^{28}$ However, as in our study, during maximum exhalation, the abdominal muscles and 
oblique muscles contract and exhalation occurs. In our study, the reason for the significant decrease in the right external oblique muscle during maximal expiration can be interpreted as an increase in efficiency due to decreased muscle activity during muscle contraction. When thoracic expansion was restricted, maximal expiration was performed using less energy as the muscle activity of the forced exhalation muscle was reduced without any difference in the respiratory parameter of maximal expiration pressure. In addition, since the external oblique muscle reduces the infrasternal angle, it can be assumed that the reduction of the external oblique muscle was not mobilized because the infrasternal angle was already reduced by applying a non-elastic belt to the rib cage. The right hand was the dominant hand in all the subjects participated in this experiment, but no relevant research results were found in the literature on whether the external oblique muscle in the dominant side leads to a decrease in the infrasternal angle compared to the non-dominant side. Also, in this study, the movement of the trunk or the movement of the limbs during the maximum respiration measurement could have an effect on the right external oblique muscle activity.

The limitations of this study are as follows: first, diaphragmatic descent and elevation could not be measured directly, and diaphragm movement was analyzed by measuring maximal inspiratory pressure, an indirect indicator. Second, since the experiment was conducted with healthy male adults, there were limitations to generalizing the results of this study. Third, only cross-sectional studies were conducted in this study. However, the inability to compare the effects of different application periods or various intervention methods may be a limitation of the study.

In conclusion, the lower rib limitation applied to prevent excessive infrasternal angle during breathing enabled the efficient use of right external oblique muscle, although it did not bring about the change of maximum expiration as expected. However, the restriction of the lower ribs caused changes in muscle use in normal subjects, it will be necessary to investigate the long-term training effect of the non-elastic belt on patients with low back pain and to measure the diaphragm movement objectively with radiography.

\section{REFERENCES}

1. De Troyer A, Estenne M. Functional anatomy of the respiratory muscles. Clin Chest Med. 1988;9(2):175-93.

2. Hruska Jr R. Influences of dysfunctional, respiratory mechanics on orofacial pain. Dent Clin North Am. 1997;41(2):211-27.

3. Panjabi MM. The stabilizing system of the spine. Part I. Function, dys- function, adaptation, and enhancement. J Spinal Disord. 1992;5(4):3839 .

4. Hodges PW, Heijnen I, Gandevia SC. Postural activity of the diaphragm is reduced in humans when respiratory demand increases. J Physiol. 2001;537(3):999-1008.

5. Hodges PW, Eriksson AM, Shirley D et al. Intra-abdominal pressure increases stiffness of the lumbar spine. J Biomech. 2005;38(9):1873-80.

6. Bye PT, Esau SA, Walley KR et al. Ventilatory muscles during exercise in air and oxygen in normal men. J Appl Physiol. 1984;56(2):464-71.

7. Loring SH, Mead J. Action of the diaphragm on the rib cage inferred from a force-balance analysis. J Appl Physiol Respir Environ Exerc Physiol. 1982;53(3):756-60

8. Boyle KL, Olinck J, Lewis C. The value of blowing up a balloon. N Am JSports Phy Ther. 2010;5(3):179-88.

9. Karvonen J, Saarelainen S, Nieminen M. Measurement of respiratory muscle forces based on maximal inspiratory and expiratory pressures. Respiration. 1994;61(1):28-31.

10. Nici L, Donner C, Wouters E et al. American Thoracic Society/European Respiratory Society Statement on Pulmonary Rehabilitation. Am J Respir Crit Care Med. 2006;173(12):1390-413.

11. Smith MD, Russell A, Hodges PW. Disorders of breathing and continence have a stronger association with back pain than obesity and physical activity. Aust J Physiother 2006;52(1):11-6.

12. Kolář P, Šulc J, Kynčl M et al. Postural function of the diaphragm in persons with and without chronic low back pain. J Orthop Sports Phys Ther. 2012;42(4):352-62.

13. Perri MA, Halford E. Pain and faulty breathing: A pilot study. J Bodyw Mov Ther. 2004;8(4):297-306.

14. Janssens L, Brumagne S, Polspoel K et al. The effect of inspiratory muscles fatigue on postural control in people with and without recurrent low back pain. Spine. 2010;35(10):1088-94.

15. Janssens L, Brumagne S, McConnell AK et al. Greater diaphragm fatigability in individuals with recurrent low back pain. Respir Physiol Neurobiol. 2013;188(2):119-23

16. Hussain SN, Pardy RL. Inspiratory muscle function with restrictive chest wall loading during exercise in normal humans. J Appl Physiol. 1985; 58(6):2027-32.

17. Van Noord JA, Demedts M, Clement J et al. Effect of rib cage and abdominal restriction on total respiratory resistance and reactance. J Appl Physiol. 1986;61(5):1736-40.

18. Petroll WM, Knight H, Rochester DF. Effect of lower rib cage expansion and diaphragm shortening on the zone of apposition. J Appl Physiol. 1990;68(2):484-8.

19. Criswell E. Cram's introduction to surface electromyography. London, Jones \& Bartlett, 2010.

20. Vera-Garcia FJ, Grenier SG, McGill SM. Abdominal muscle response during curl-ups on both stable and labile surfaces. Phys Ther. 2000; 80(6):564-9.

21. Kendall FP, McCreary EK, Provance PG et al. Muscles testing and function with posture and pain. 5th ed. Baltimore, Williams \& Wilkins, 2005: 202-7.

22. Klefbeck B, Hamrah Nedjad J. Effect of inspiratory muscle training in patients with multiple sclerosis. Arch Phys Med Rehabil. 2003;84(7): 994-9. 
23. Kim JH, Part HJ, Jeon SY et al. Initial effect of an elastic chest band during inspiratory exercise on chest unction improvement in people with limited rib mobility: A randomized controlled pilot trial. Physiother Res Int. 2012;17(4):208-13.

24. Larson JL, Covey MK, Vitalo CA et al. Maximal inspiratory pressure. Learning effect and test-retest reliability in patients with chronic obstructive pulmonary disease. Chest. 1993;104(2):448-53.

25. Truwit JD, Marini JJ. Validation of a technique to assess maximal inspira- tory pressure in poorly cooperative patients. Chest. 1992;102(4):1216-9. 26. Smith M, Ball V. Cardiovascular/Respiratory physiotherapy. London, Mosby, 1998:4-5.

27. Korean Academy of Cardiorespiratory Physical Therapy. Cardiovascular and Pulmonary Physical Therapy Intervention, Seoul, PanMun, 2014: 165-6.

28. Neumann DA. Kinesiology of the musculoskeletal system. Foundations for rehabilitation. Philadelphia, Mosby Elsviere, 2010:440-8. 